\title{
Primary Orbital Cystic Pilocytic Astrocytoma
}

\author{
Hardeep Singh Mudhar ${ }^{\mathrm{a}}$ Murtuza Nuruddin ${ }^{\mathrm{b}}$ Soma Roy ${ }^{\mathrm{b}}$

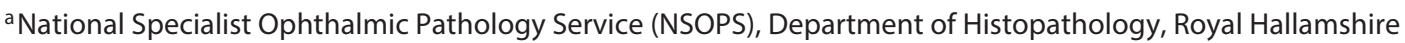 \\ Hospital, Sheffield, UK; ${ }^{b}$ Chittagong Eye Infirmary and Training Complex, Chittagong, Bangladesh
}

\section{Key Words}

Pilocytic astrocytoma $\cdot$ Brain heterotopia $\cdot$ Orbit · Primary

\begin{abstract}
Purpose: To describe the clinical, radiological and histopathological features of a highly unusual primary orbital glioma. Procedures: A 6-year-old female presented with right-sided proptosis that revealed a well-defined intraconal mass on $\mathrm{CT}$ imaging with intact orbital bony walls. During the removal of the lesion, it dissected away freely from the muscles and was separated from the optic nerve and globe. There was no communication with the brain. Results: Histology showed histological features highly favouring a pilocytic astrocytoma enveloped by meninges. No non-neoplastic brain tissue was identified. Conclusion: This is the first documentation of a primary orbital pilocytic astrocytoma not anatomically connected with the brain. We speculate that the tumour had arisen from developmental brain heterotopia.
\end{abstract}

C 2016 S. Karger AG, Basel

\section{Introduction}

Pilocytic astrocytomas can affect the visual pathway mainly in the paediatric age group. In the orbit they arise in the optic nerve and tend to be dura-bound fusiform swellings with distinctive imaging and histological characteristics. They often extend into the optic chiasm and brain and may be associated with neurofibromatosis type [1-3]. We present the clinical, radiological and histopathological features of a highly unusual case of glioma in a 6-year-old female who presented with right-sided massive proptosis; during surgical exploration the tumour was intraconal and was separated from the optic nerve, globe and extraocular muscles and not connected to the brain.

\section{Case Report}

A 6-year-old female presented to the Orbit \& Oculoplastic Clinic of the Chittagong Eye Infirmary with increasing protrusion of the right eye over a 3-year period (fig. 1a). There was no history of trauma or any serious systemic diseases. Her prenatal, natal and postnatal history was uneventful. There were no stigmata of neurofibromatosis. On examination there was no perception of light in the right eye. There was severe proptosis with downward and lateral displacement of the globe along with exposure keratopathy (fig. 1a). Ocular motility was restricted in all gazes. The anterior and posterior segments were not visible. On palpation, there was a firm, non-tender mass behind the globe, extending into the deep orbit. Examination of the left eye was unremarkable.

A CT scan showed a well circumscribed isointense, cystic, intraconal lesion, pushing the globe forward. There was no intracranial extension of the lesion and it was not associated with any orbital bony defect, erosion or hyperostosis (fig. 1b-e). The patient

\section{KARGER}

E-Mail karger@karger.com

www.karger.com/oop
(C) 2016 S. Karger AG, Base

2296-4681/16/0024-0280\$39.50/0
Dr. Hardeep Singh Mudhar

National Specialist Ophthalmic Pathology Service (NSOPS) Department of Histopathology, Royal Hallamshire Hospital Glossop Road, Sheffield S10 2JF (UK)

E-Mail hardeep.mudhar@sth.nhs.uk 

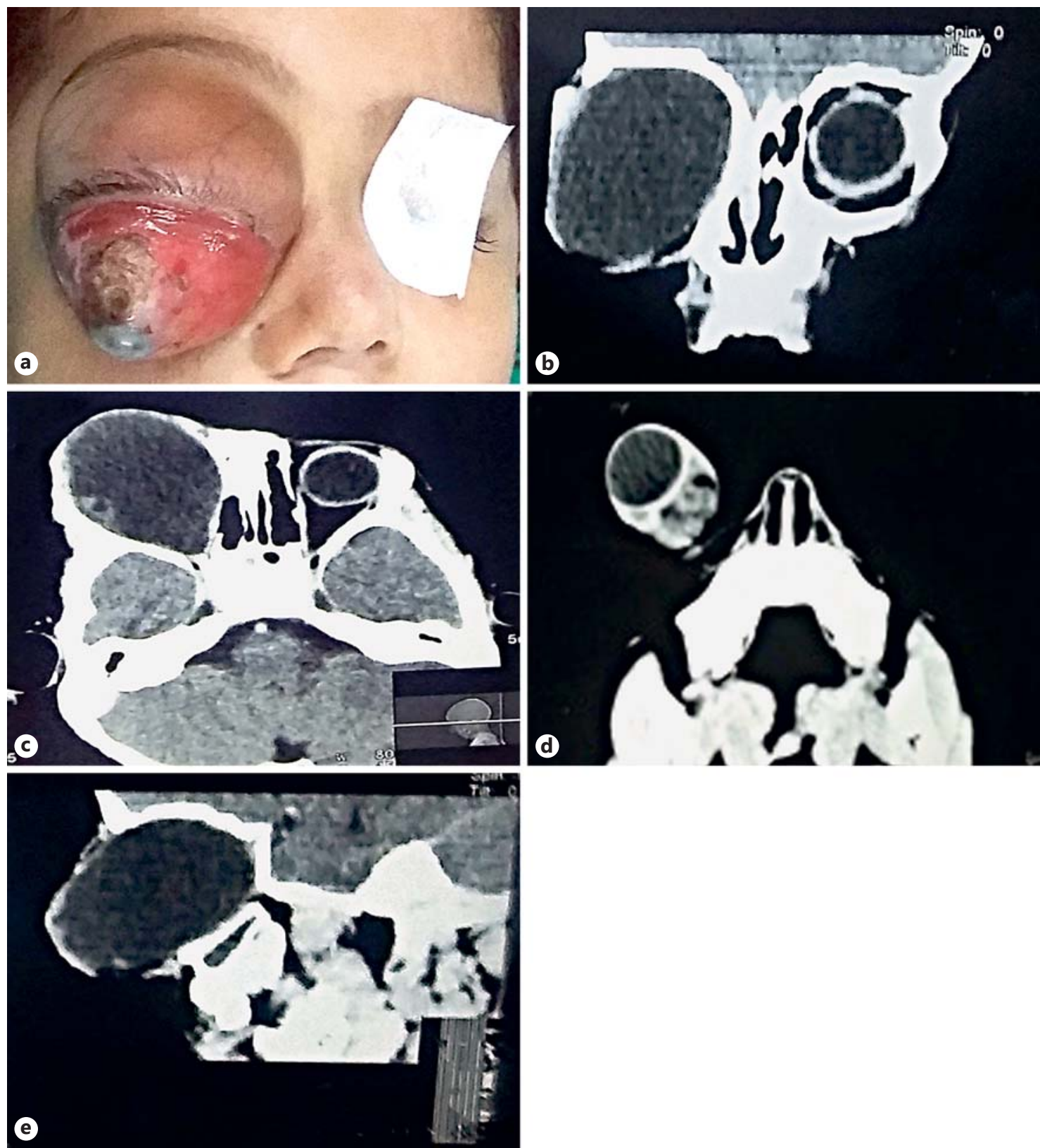

Fig. 1. a Clinical photograph showing right-sided proptosis. b Coronal CT scan section showing the right-sided mass. Note the intact orbital bones superiorly and medially. c Horizontal CT section showing the same mass on the right with intact orbit bones. d Horizontal CT section inferior to C showing the proptosed globe. e Sagittal $\mathrm{CT}$ section illustrating the mass and the intact orbit bones. Note the normal optic nerve tract posteriorly.

proceeded to surgical excision. During surgery, clear fluid was drained from the cyst followed by complete excision. The lesion was separated from the globe, optic nerve and extraocular muscles and there were no defects affecting the bony orbit and no communication with the brain.

The specimen sent to the ophthalmic pathology laboratory was a collapsed grey-coloured lesion, $45 \mathrm{~mm}$ in maximum dimension, with a smooth external surface. The cut surface revealed a cystic cavity containing gelatinous brown contents, lined by a solid, cream-coloured wall up to $8 \mathrm{~mm}$ in maximum thickness (fig. 2a). Histology of the lesion showed a complete external layer of dura mater (fig. 2b) and arachnoid with meningothelial hyperplasia (fig. 2c). At scanning power, the lesion breached the dura (arrows in fig. 2d). At higher power, the lesion was composed of a moderately cellular lesion comprising a mixture of multipolar and piloid astroglial cells (fig. 2e). In some areas the astroglial cells were ar- 
a

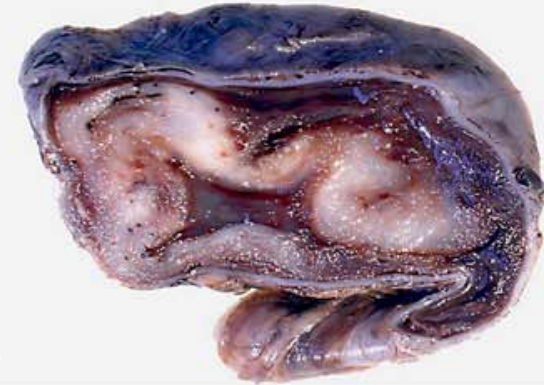

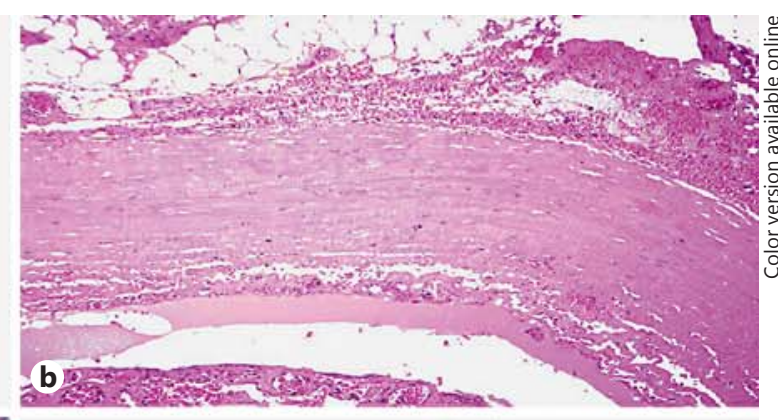

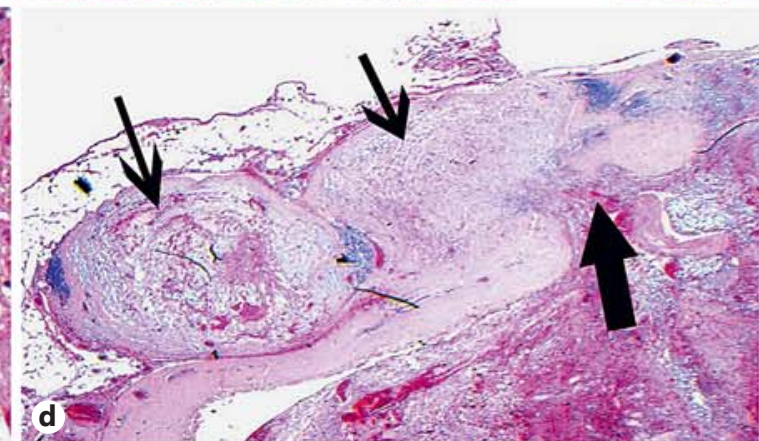

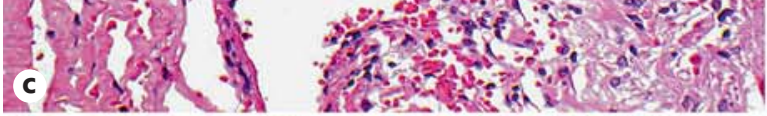

19.

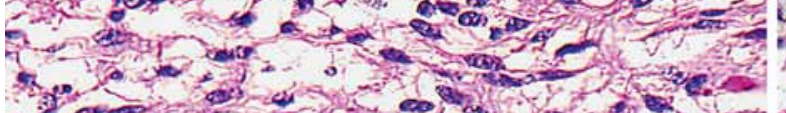

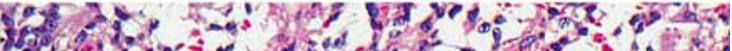
Hof

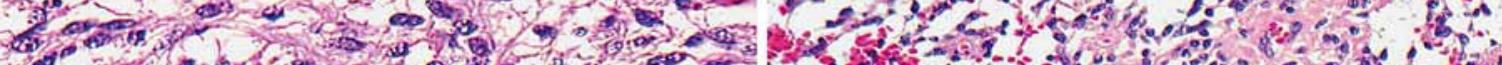

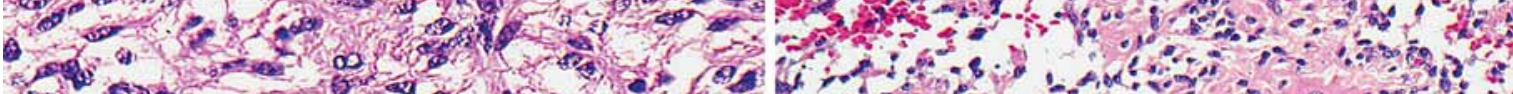

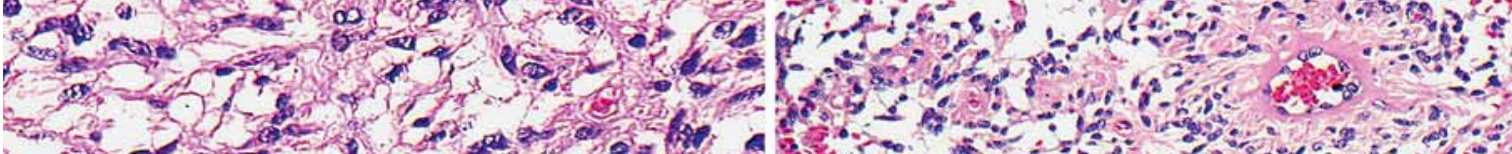

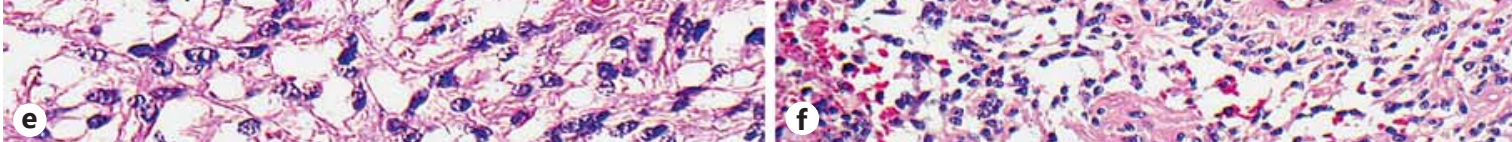
W

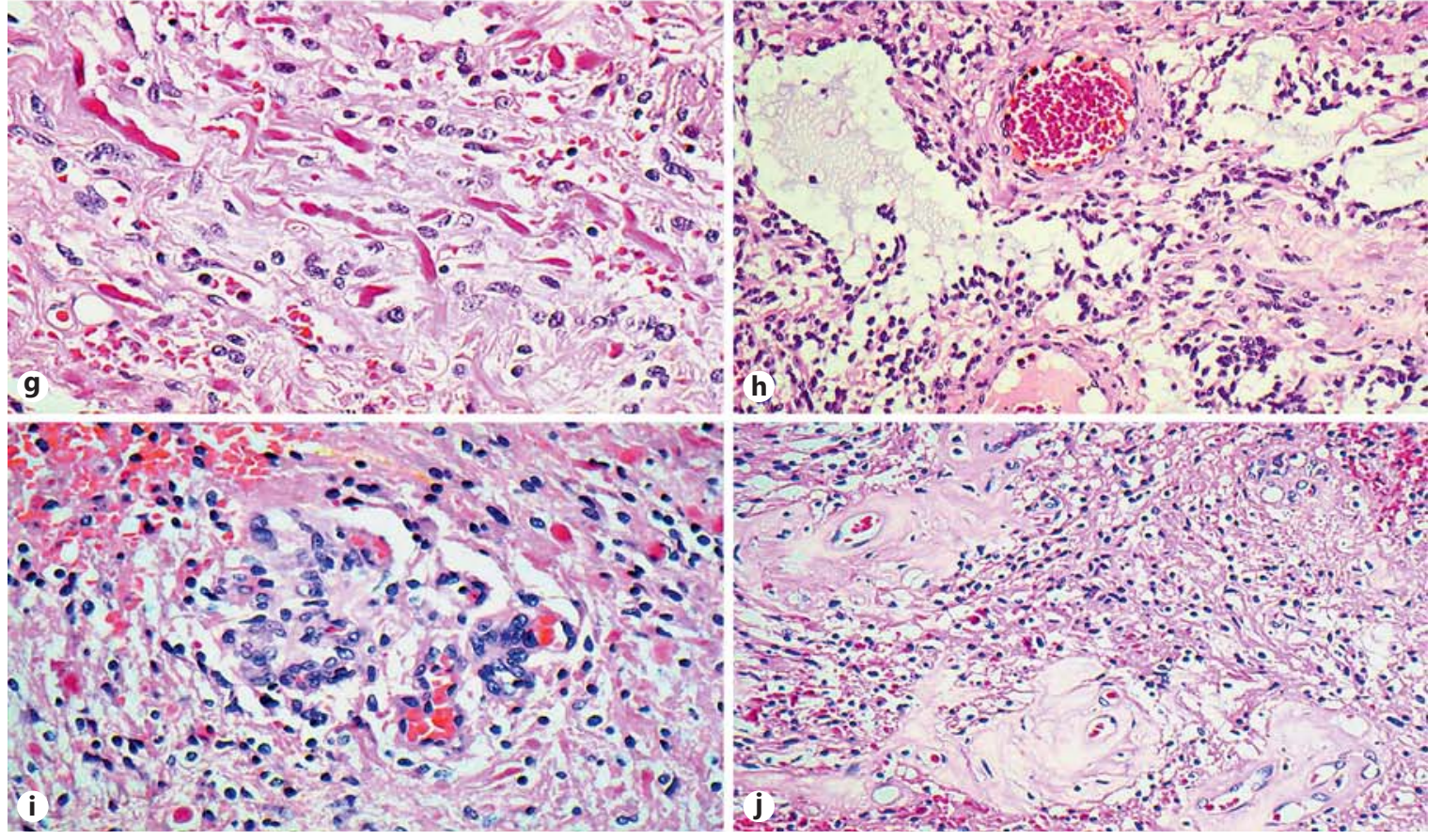

(For legend see next page.) 
ranged in a biphasic pattern with perivascular condensation alternating with looser areas (fig. 2f) and myxoid 'cysts' (fig. 2g). Numerous Rosenthal fibres were seen, especially in the more compact areas with piloid cells (fig. $2 \mathrm{~h}$ ). The lesion contained glomeruloid vasculature (fig. $2 \mathrm{i}$ ) and vascular hyalinisation regressive changes (fig. 2j). The central part of the lesion was filled with proteinaceous exudate with recent and old haemorrhage (not shown). No 'normal' brain tissue was identified (i.e., no neuronal tissue was present). No choristomatous elements were mixed in with the tumour. The astroglial cells in the lesion were strongly positive for GFAP (not shown). Ki67 showed a $<1 \%$ proliferation fraction amongst the astroglial cells (not shown). The tumour was negative for isocitrate dehydrogenase 1 (IDH1-R132H) by immunohistochemistry (not shown). Molecular analysis showed no evidence of KIAA1549-BRAF fusion and no mutations in BRAF V600E were identified. Following surgery, the proptosis markedly resolved and the postoperative course was uneventful.

\section{Discussion}

The primary question in such cases is whether the lesion represents an extensive piloid gliosis or whether it is a pilocytic astrocytoma. Making the distinction in this case was complicated by negativity for KIAA1549-BRAF fusion and the presence of wild-type BRAF V600E. These molecular alterations are classically found in a high proportion of pilocytic astrocytomas $[4,5]$, but not in every case. Furthermore, the lesion was negative for IDH1$\mathrm{R} 132 \mathrm{H}$ by immunohistochemistry. IDH1 is classically expressed by non-pilocytic astrocytomas [6] although a few exceptions exist. Histological parameter assessment was therefore used to make the distinction. There are certain morphological features that are common to piloid gliosis and pilocytic astrocytoma, such as the presence of Rosenthal fibres, hypercellularity and bipolar astroglial cells. However, the most compelling evidence supporting a neoplasm in our case was the degree of biphasic hypercellularity and microcystic arrangement of the cells around vessels and glomeruloid-type vascular proliferation. Fur-

Fig. 2. a Macroscopic photograph of the cut surface of the mass, showing convoluted thickening of the wall by a greyish/creamcoloured lesion, surrounded by meninges and a cystic cavity. b-j Haematoxylin and eosin-stained sections. b External dural lining. c Meningothelial cell hyperplasia (arrow). d Extradural invasion (thin arrows) through the dura (thick arrow). e The tumour was composed of stellate and bipolar bearing astroglial cells. $\mathbf{f} \mathrm{Bi}$ phasic histology with perivascular condensation of tumour cells alternating with looser areas. $\mathbf{g}$ Numerous Rosenthal bodies. $\mathbf{h} \mathrm{Mi}$ crocystic tumour areas. i Glomeruloid vascular changes. j Sclerotic tumour vessels.

Primary Orbital Cystic Pilocytic

Astrocytoma thermore, the lesion was invading through a dural defect into the orbital fat, attached to the surface of the lesion. These features are not usual in piloid gliosis, and it is for these reasons that the lesion in question was judged to be a pilocytic astrocytoma rather than piloid gliosis.

There are two possible explanations for the origin of the tumour. One possibility is that brain heterotopia occurred during development, with subsequent sequestration and anatomic discontinuity [7-10]. This would have been followed by tumour development with effacement of all heterotopic brain tissue. A second possibility is mesenchymal stem cells giving rise to the tumour. The presence of a complete layer of meninges and a cystic cavity would favour a developmental out-pouching of embryonic brain rather than a mesenchymal stem cell derivation. It is interesting to note that the glioma was 'invading' the surrounding orbital fat through a dural defect. It is worth speculating whether this defect may have represented the point at which the heterotopic tissue was tethered to the rest of the brain before losing its anatomic connection.

There have been several previous case reports documenting heterotopic/ectopic brain in the orbit [7-10], but none of those displayed features of a pilocytic astrocytoma. We examined the whole specimen as several slices and could not detect any non-neoplastic brain tissue with calcospheres (commonly observed in heterotopic brain) [7-10].

Two publications in the literature have highlighted the difficulty in establishing the true nature of glial proliferations in heterotopic locations. Bruzek et al. [11] reported a 'pilocytic astrocytoma' that focally arose within an encephalocele, arising from herniation of the frontal lobe into the superior orbit. The patient was a 29 -year-old male who presented with seizures. There was a prior history of trauma. Examination showed mild right-sided proptosis, and an MRI scan revealed herniation of the frontal lobe into the orbit with globe and superior rectus muscle displacement inferiorly. The encephalocele was excised and showed neuroglial tissue with a ' $0.5 \mathrm{~cm}$ focus of low-grade pilocytic astrocytoma encased by the encephalocele with no involvement of the optic pathway'. The diagnosis of pilocytic astrocytoma was based on the presence of Rosenthal fibres, hypercellularity and involvement of the leptomeninges, without molecular confirmation. The authors did discuss the possibility of piloid gliosis occurring in an encephalocele, especially given the history of trauma, but opted to designate the lesion as neoplastic, based purely on leptomeningeal involvement, which is unusual in a reactive glial proliferation. Our case

Ocul Oncol Pathol 2016;2:280-284 
did not occur in the context of an established encephalocele but in probable sequestered brain tissue. There was no history of trauma. Secondly our case showed more convincing histological features supporting a glioma, such as the presence of biphasic histology with microcytic areas and glomeruloid vasculature, than the features illustrated in that publication.

A further publication by Ellouze et al. [12] documented a right frontonasal mass in a newborn that was detected on a routine 24-week gestation ultrasound. An MRI at 27 weeks gestation showed an extracranial frontonasal mass and an associated intracranial mass anterior to the frontal dura, without continuity between the two components. A postoperative 3D CT scan showed a 5-mm defect in the right nasal bone. The child underwent excision of the right dermal frontonasal mass and the intracranial lesion. The dermal frontonasal mass revealed an astroglial proliferation invading the dermis, suggestive of an encephalocele. The intracranial component showed bipolar astroglial cells without mitotic activity associated with scattered calcific bodies and a few vessels with thickened walls. The authors speculated whether this was a gliosis or a low-grade pilocytic astrocytoma. The lesion was negative for IDH1 on immunohistochemical testing and had a very low Ki67 proliferation index. It is interesting to note that the authors did not consider the intracranial part to be just brain heterotopia with gliosis, given the presence of calcifications that are common in brain heterotopias as alluded to. Secondly, there was no orbital component in that case report, and features such as a biphasic cytology, microcytic hypercellular areas and glomeruloid vessels were completely absent.

Another diagnosis meriting consideration includes a congenital cystic eye and microphthalmia with cyst [10]. However, this can be confidently excluded by the presence of an anatomically intact globe at birth and during the surgical procedure.

In summary, we have presented the clinical, radiological and histopathological features of a unique case of a primary intraconal orbital pilocytic astrocytoma not connected to the optic nerve or brain that was successfully treated by complete excision.

\section{Statement of Ethics}

The subject's parents gave their informed consent for publication of this case report (text and photographs). The study protocol was approved by the institute's committee on human research. The study involved no animal experimentation.

\section{Disclosure Statement}

This study did not attract specific sponsorship or funding. There are no conflicts of interest.

\section{References}

1 Dutton JJ, Byrne SF, Proia AD: Diagnostic Atlas of Orbital Diseases. Philadelphia, WB Saunders, 2000.

2 Shields JA, Shields CL, Scatozzi R: Survey of 1264 patients with orbital tumors and simulating lesions: the 2002 Montgomery Lecture, part 1. Ophthalmology 2004;111:997-1008.

3 Czyzyk E, Jozwiak S, Roszkowski M, Schwartz RA: Optic nerve gliomas in children with and without neurofibromatosis 1 . J Child Neurol 2003; $18: 471-478$.

4 Jones DTW, Kocialkowski S, Liu L, Pearson DM, Bäcklund LM, Ichimura K, Collins VP: Tandem duplication producing a novel oncogenic BRAF fusion gene defines the majority of pilocytic astrocytomas. Cancer Res 2008; 68:8673-8677.

5 Schindler G, Capper D, Meyer J, Janzarik W, Omran H, Herold-Mende C, Schmieder K, Wesseling P, Mawrin C, Hasselblatt M, Louis
DN, Korshunov A, Pfister S, Hartmann C, Paulus W, Reifenberger G, von Deimling A: Analysis of BRAF V600E mutation in 1,320 nervous system tumors reveals high mutation frequencies in pleomorphic xanthoastrocytoma, ganglioglioma and extra-cerebellar pilocytic astrocytoma. Acta Neuropathol 2011; 121:397-405.

6 Parsons DW, Jones S, Zhang X, Lin JC, Leary RJ, Angenendt P, Mankoo P, Carter H, Siu IM, Gallia GL, Olivi A, McLendon R, Rasheed BA, Keir S, Nikolskaya T, Nikolsky Y, Busam DA, Tekleab H, Diaz LA Jr, Hartigan J, Smith DR, Strausberg RL, Marie SK, Shinjo SM, Yan H, Riggins GJ, Bigner DD, Karchin R, Papadopoulos N, Parmigiani G, Vogelstein B, Velculescu VE, Kinzler KW: An integrated genomic analysis of human glioblastoma multiforme. Science 2008;321: 1807-1812.
7 Wilkins RB, Hofmann RJ, Byrd WA, Font RL: Heterotopic brain tissue in the orbit. Arch Ophthalmol 1987;105:390-392.

8 Newman NJ, Miller NR, Green WR: Ectopic brain in the orbit. Ophthalmology 1986;93: 268-272.

9 Elder JE, Chow CW, Holmes AD: Heterotopic brain tissue in the orbit: case report. Br J Ophthalmol 1989;73:928-931.

10 Scheiner AJ, Frayer WC, Rorke LB, Heher K: Ectopic brain tissue in the orbit. Eye 1999;13: 251-254.

11 Bruzek A, Shepherd D, Gompel JV, Jentoft M: Pilocytic astrocytoma presenting as an orbital encephalocele: a case report. Case Rep Neurol 2015;7:90-94.

12 Ellouze N, Born J, Hoyoux C, Michotte A, Retz C, Tebache M, Piette C: Nasal heterotopia versus pilocytic astrocytoma: a narrow border. Neurochirurgie 2015;61:279-282. 